\title{
Potentials of proton magnetic resonance techniques in Radiotherapy procedures: A review
}

\author{
${ }^{1 *}$ Aweda M. A., ${ }^{2}$ Awojoyogbe O. B. and ${ }^{2}$ Dada M. \\ ${ }^{1}$ Department of Radiation Biology and Radiotherapy, College of Medicine of the \\ University/Lagos University Teaching Hospital, Idi-Araba. P.M.B. 12003, \\ Lagos State, Nigeria. \\ ${ }^{2}$ Department of Physics, Federal University of Technology, Minna, Niger State, Nigeria. \\ *Corresponding author's email: maaweda@cmul.edu.ng
}

\begin{abstract}
The current trend in technological Radiotherapy (RT) developments demands highly precise tumour volume definition and treatment simulation for satisfactory quality management. To achieve this requires sophisticated diagnostic imaging. Magnetic Resonance Imaging (MRI) is systematically emerging to break the monopoly of Computerized Tomography (CT) in treatment planning (TP) procedures by introducing several imaging benefits over CT. Such benefits include improved soft tissue definition, unlimited multi-planar, volumetric imaging, physiological and biochemical information using MR angiography and spectroscopy. Intensity-modulated and adaptive RT techniques may greatly benefit from the functional MRI. New MRI techniques can characterize and quantify different tissue properties and tumour-related changes better than CT. Perfusion MR images represent micro-vascular density and permeability, MR spectroscopy depicts specific metabolites, diffusion-weighted imaging shows tissues at risk and tumour cells while dynamic 3-D acquisition and 4-D images show organ motions and the mobility. The review of these new features, the emerging technological developments of the anatomy of MR Imager and the new contrast materials with a view to using MRI alone for RT treatment planning and other RT procedures form the object of this paper. Literature, internet facilities, relevant documentary and private communications were the sources of the review.
\end{abstract}

Keywords: Magnetic Resonance Imaging, Radiotherapy, Treatment Planning, Patient Monitoring.

\section{INTRODUCTION}

Radiotherapy (RT) as one of the major modalities for cancer management involves simulation and planning of the patient treatment. The facilities used range from radiography, conventional tomography, $\mathrm{x}$ ray simulator to computerized tomography (CT) and Magnetic Resonance Imaging (MRI). The recent advances in MRI promote its use in cancer management. Our recent studies of the subject shows that MRI techniques and technology are youngest and the fastest growing of all medical diagnostic modalities (Dada et al. 2010, Awojoyogbe et al. 2010a, Awojoyogbe et al. 2010b ); the new and developing contrast materials and the recent keener interest of radiological scientists necessitate a review of the subject, which is the aim of this paper using recent publications, internet facilities, relevant documentary and private communications.

Relevance of MRI in cancer management: CTbased 3-D treatment planning systems have been routinely used in most RT Departments. MRI has today emerged to break this monopoly of CT as the most popular RT simulator. MRI successfully complements CT-based TP for some patient anatomical parts (Parke and Patrocinio 2005); both provide more consistent target volume definitions than with either MRI or CT alone (Mizowaki 2002). RT techniques such as intensity-modulated radiation therapy (IMRT) (Chao et al. 2008) and lately SingleArc IMRT (Bortfeld and Webb 2009), allow for better and more precise dose distribution within the target volume while sparing a larger portion of healthy tissues than conventional RT. These techniques require more highly precise tumour volume definition in TP. MRI applications today go beyond TP to other RT processes due to some benefits over other modalities such as superior soft tissue definition, unlimited multi-planar volumetric imaging, physiological and biochemical information using magnetic resonance angiography (MRA) and magnetic resonance spectroscopy (MRS). The recent 
open and low magnetic field MR simulator (Krempien et al. 2003 Mack et al. 2005) provides easier integration for planning. Ultra-small superparamagnetic iron oxide (USPIO) contrast agents can today be used to evaluate pathological lymph nodes treatment (Weissleder et al. 1990). Ultra-fast volumetric and cine mode sequences can provide temporal assessment of target volume deformity and positioning for IMRT (Plathow et al. 2004). High intensity field scanners provide higher resolution images for better tissue definition and can also benefit MRS applications (Payne and Leach 2006, Louis et al. 2007). Techniques using dynamic contrast-enhanced MR (Krauss et al. 2005 and Sanghvi 2009), diffusion weighted MR and Diffusion Tensor Imaging (Dzik-Jurasz et al. 2002, Kremser et al. 2003) can further define target volumes with improved and complementary morphological, functional and biological data. The techniques may also be combined with positron emission tomography (PET) to further increase the diagnostic sensitivity and specificity (Podgorsak et al. 2005, Hrabe et al. 2007). MRI is now being increasingly used in oncology not only for treatment planning, but also for staging, assessing tumor response and evaluating disease reoccurrence. As a result of the enhanced image properties and qualities, MRI is becoming a more cost effective diagnostic tool in the managing cancers and some other diseases (Mackenzie and Dixon 1995, Khoo 2000). Some new and developing techniques, new contrast materials and new MRI anatomical and methodological techniques are reviewed to assess their current and future potentials for applications in RT procedures. MRI systems not using ionizing radiation is a strong attraction to radiologists and comfort to patients just as contrast materials have recorded low toxicity (Li et al. 2008).

\section{MRI IN CANCER DIAGNOSIS AND STAGING}

The major steps in patient RT management procedure are illustrated in Figure 1; starting with cancer diagnosis through patient data acquisition to RT delivery. Since its initial clinical use in the early 1980s, MRI has become an important modality for diagnosis of diseases (Los Alamos National Laboratory 2007). Diagnostic and staging potentials are boosted by the recently emerged contrast materials. An assessment of USPIO for example, showed high sensitivity and specificity of up to $90 \%$ of cases with small volume tumor nodal involvement (Harisinghani et al. 1999, Mack et al. 2002). This contrast method with 3-D sequences can provide visualization along blood vessels and allow mapping of lymph nodes (Portaluri et al. 2004). In its application USPIO particles are injected and taken up by macrophages and transported via the lymphatic system to the nodes. In the reticulo-endothelial tissues of normal lymph nodes the ingested particles produce a reduction in signal intensity within the node due to the negative enhancement from the iron oxide particles. USPIO imaging permits individualization of treatment fields as exemplifies in the management of head and neck planning of surgery (Martin et al. 2005). It can also complement the current recommendation for CT-defined nodal volumes for head and neck RT (Gregoire et al. 2000). The threshold size for detecting pathological involvement of nodes and false positives may be detected if there is fibrosis or fatty replacement of nodes. It may be combined with other imaging methods such as PET to further increase sensitivity and specificity (Solberg et al. 2004).

MRI scanning for tissue volume definition: Tumor volume definition is critical; poorly defined volume may result in exclusion of some tumor cells out of primary radiation beam. Precise localization of the target volume and its relationship with the surrounding tissues are essential for quality RT management. Some important measures for optimizing MR image quality and tissue volume definitions include:

(a) Adjustment of Imaging Parameters: The contrast from tissue structures can be widely varied by adjusting the imaging parameters like proton densities and relaxation times $T_{1}$ and $T_{2}$. This flexibility allows improved characterization of tissues even when they possess similar electron densities. MR sequences give better tissue discrimination between the tumor volume, its boundaries and adjacent normal tissues. Sequences apply not only to the initial RT treatments but also for re-treatments based on ability to differentiate between changes in recurrent cancer and secondary post-treatment fibrosis (Seddon et al 2003). Segmentation algorithms for MRI have recently been developed for target volume definition based on the contrast enhancement ratio of $\mathrm{T}_{1}$-weighted images and signal intensity of $\mathrm{T}_{2}$-weighted images (Partridge et al 2006). In the pelvis, MRI has provided improved target delineation for gastrointestinal, urological and gynaecological cancers. For prostate cancer, it provides better image of the internal organs than $\mathrm{CT}$ for disease spread, capsular and seminal vesicle involvement (Watanabe et al. 2005, Mizowaki et al. 1996). It overcomes some of the limitations of CT in 
the definition of prostate target volumes. MR images, particularly sagittal views, are useful in defining the prostatic apex and distinguishing between the boundaries of the prostate with the base of the bladder and the anterior wall of the rectum (Mah et al. 2002, Lee et al. 2003). Comparative MRI and CT planning studies using MRI-defined prostate volumes as standard have shown that CT-defined prostate volumes tend to overestimate target volumes by as much as $27-43 \%$ due to the soft tissue uncertainty (IPEM 1995, AAPM 1990, Price et al. 1990, Och et al. 1992).

(b) Manipulation of MR Sequences: The manipulation of proton relaxation times provides the two basic $T_{1}$ - and $T_{2}$ - weighted MR sequences. While this allows better imaging of soft tissues, parameters may as well be manipulated to benefit TP by providing ultra-fast imaging, volumetric sequences and cine-mode acquisition. These will provide information on target motion and critical organ displacement (Huerlimann 2001). A series of ultrafast imaging may be obtained by sequences such as echo-planar imaging. MRI better defines structures such as myocardium and ventricular space compared with CT for heart dose-volume assessment by breath hold for left breast RT (Pickett et al. 1999). Ultra-fast acquisitions may also be used to obtain gated images to model organ and target motions (Rohlfing et al. 2004). Sequential volumetric and cine-mode acquisitions can benefit IMRT by providing data for the implementation of appropriate site-specific planning margins for both the target and critical organ. Cine-MRI can evaluate intra-thoracic tumor mobility for patient individualization of treatment margins and assessment of the efficacy of free breathing gating techniques in lung RT (Plathow et al. 2004).

(c) Increase of magnetic field strength: The quality of the image increases with the signal strength as the signal-to-noise (SNR) is increased. SNR increases approximately linearly with magnetic field, The magnetic field strength of scanners commonly used in clinics is about 0.2 to $0.5 \mathrm{~T}$, but recently, advances have been achieved through introduction of $1,1.5,3$ and even $7 \mathrm{~T}$ to obtain improved image quality (Liu et al. 2004). In prostate MRI, the image quality from external phased array coils using $3 \mathrm{~T}$ is comparable with endorectal coils at $1.5 \mathrm{~T}$ (Bloch et al. 2004) and in some cases exceeding them (Barentsz et al. 1999). 3 T MRI can also benefit the application of functional MRI (fMRI) and MRS by providing better resolution for the studied metabolites that define tumor regions as in the brain and prostate RT (Sosna et al. 2004). 3 T MRI uses higher field gradient systems and requires increased shielding with smaller scanner bores. The small bores restrict the size of the patient scanned while the high fields may increase the potential for patient claustrophobia. A 7 $\mathrm{T}$ MRI has been employed in advanced diagnosis and treatment of brain diseases and cancers. Figure 1 compares the same anatomical part with $3 \mathrm{~T}$ (left) and with $7 \mathrm{~T}$ (right) in which ultra-high magnetic field strength greatly enhances image resolution and enables high-speed measurements (National German Metrology Institute 2009).

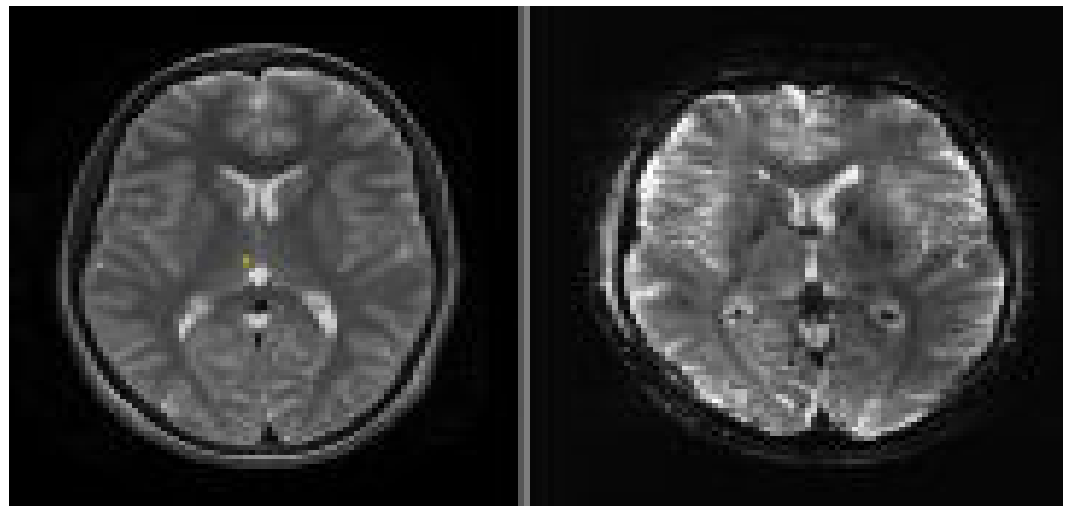

Fig 1: Resolution comparison of $3 \mathrm{~T}$ and $7 \mathrm{~T}$ magnetic field images

(d) Open and low magnetic field MR: Open and low field scanner has some advantages including greater $\mathrm{T}_{1}$ image contrast, reduced vessel flow, ghost artifacts and the possibility of using patient immobilization devices limited by size in the conventional MRI scanners. Although it has higher system-related distortions which are possible to compensate for, patient-induced distortions are 
significantly less due to the lower field strength (Krempien RC et al. 2003, Mack et al. 2005).

(e) Water Diffusion technique: MRI utilizes the changing mobility of water molecules to obtain wide range of variable image contrasts, complemented by the use of a range of contrast agents (Le Bihan et al. 1986). Spatial resolution with typical range 0.5-2.0 $\mathrm{mm}$ is traded for signal-to-noise ratio (SNR) and the scan time with slice thickness which typically ranges from $0.5-7.0 \mathrm{~mm}$. Images are usually obtained as 2-D sets of slices or as 3-D volume acquisitions, and although usually obtained parallel or trans-axial to the magnet axis, they can generally be obtained at any arbitrary plane.

(f) Diffusion Tensor Imaging Technique: Diffusion tensor (DT) MRI shows white matter abnormalities based on cerebral tissue anisotropy. Tissue disorganization provides information about brain tumor involvement on white matter tracts. The method can be used for assessing white matter infiltration by occult tumor (Dzik-Jurasz et al. 2002, Kremser et al. 2003). DT techniques recorded larger white matter tract abnormalities than those found on $\mathrm{T}_{2}$-weighted images in 10 out of 13 high-grade gliomas and previously unrecognized contra-lateral involvement in 4 of these cases (Dzik-Jurasz et al. 2002). A study further suggested that treated volumes may be optimized using this technique by reducing the target volume compared with CT planning alone, thus providing the opportunity of dose escalation whilst maintaining tissue tolerance (Price et al. 2003). Magneto-encephalography and DT techniques can also be used to limit doses to relevant functional regions of the cerebrum and white tracts to reduce specific radiation-induced neurological dysfunction (Hrabe et al. 2007). The technique also offers complementary information with other imagers including PET.

(g) MR Spectroscopy: MRS is a noninvasive method that identifies various metabolites on the basis of their slightly different chemical shifts or resonance frequencies. MRS can help to differentiate between recurrent tumors and changes in brain tissue due to radiation treatments. Manganas et al. (2007) recently described a metabolic biomarker for the detection and quantification of neural progenitor cells in the brain in-vivo using MRS. They demonstrated its use as reference for monitoring neurogenesis. Applications have opened opportunity for investigating the role of neural progenitor cells and neurogenesis in brain disorders. MRS may also be used to determine the concentrations of metabolites,
Choline, creatine and $\mathrm{N}$-acetylaspartate which are commonly investigated. These techniques, together with spectroscopic mapping aid definition of tumor limits (Khoo et al. 2000). This is useful for diagnosing certain metabolic disorders that affect the brain as well as provide information on tumor metabolism (Rosen et al. 2007).

\section{MRI IN TREATMENT PLANNING AND SIMULATION}

The MRI-simulation scan is similar to conventional diagnostic scans. The primary differences are the requirements for patient positioning, immobilization, treatment specific scan protocols, often increased scan limits, use of contrast, placement of localization marks on the patient skin, and some other special considerations.

(a) Patient data acquisition: Patient data acquisition generally involves direct measurements of anatomical parts on the patient and the use computer-assisted TP systems. The computerized systems make it possible to outline the external shapes in all areas where the radiation beam enters and exits the patient. The adjacent areas and internal structures are also outlined to determine shapes and volumes for dose calculations. Some unforeseen problems with patient set-up for the chosen treatment technique may be solved through simulation, thereby saving some of the time to be spent in the treatment room. Apart from tumor localization and clinical beam setup, other necessary patient data may be obtained during simulation. MRI scans provide the information required for complex TP, one of the basis for their use in modern RT (Khoo et al. 2006, Rasch et al. 1999, Pech et al. 2008). Since the first production of a blurry image from MRI scan in 1977 (Damadian et al. 1977, Hinshawet al. 1977), MRI has greatly improved to produce high-quality images not well demonstrated with $\mathrm{x}$-ray-based imagers.

(b) Treatment setup and simulation: The simplest form of simulation involves the use of portal films obtained on the treatment machine to establish the geometry and setup, although this is not an efficient and practical approach. Treatment machines operate using $\mathrm{MeV}$ and not keV range of energies and, therefore, do not produce good quality radiographs. Secondly, the machine is used for treatment, the use of same for simulation is not convenient. Simulation typically consists of contouring of the target and normal structures, placement of the treatment isocenter and the beams, design of treatment portal shapes, generation of digitally reconstructed 
radiographs (DRRs) and documentation. Methods for simulating specific treatment sites have been described by several authors (Payne and Leach 2006, Khoo et al. 1999, Newbold et al. 2006, Heester et al. 1993). MRI simulation process begins with target and normal volume delineation. Other imaging studies such as CT, US, PET may be registered and/or fused to the MRI-scan to provide information for improved target or normal tissue delineation or for the purpose of dosimetry, especially with CT. The setup marks are placed on a stable anatomical location and then shifts are applied to the treatment isocenter for every treatment.

(c) Patient setup, placement of beams and design of treatment portals: The patient is setup according to instructions from the MRI-simulation software. Port films are acquired and compared with MRI-simulation DRRs. In some cases, the patient may undergo treatment setup verification on a conventional simulator prior to the treatment. This can be valuable for treatment sites in the thorax and abdomen for example, due to the MRI inability to display breathing motion. A well-designed CT-simulation process can cause all of these steps to appear relatively seamless, and the duration of the entire process relatively short. MRI- and CT-simulation data images, contours and treatment beams are then communicated to TP system equipped with dose calculation software.

\section{POTENTIALS ROLES OF MRI IN RT TREATMENT PROCESSES}

Applications of MRI go beyond imaging for diagnosis, definition of target volumes and RT treatment simulation. There is increasing dependence of advanced RT techniques on image guidance which plays a primary role in CNS disease management and others like prostate (Kremser et al. 2003), head and neck cancers (Sultanem et al. 2004). A number of other new applications during RT course have emerged while some are still undergoing studies. Some of these innovations are briefly discussed below.

(a) Diffusion-weighted MR sequence: Diffusionweighted MR sequence is a pulse sequence sensitized to the random Brownian motion of water molecules (Krauss et al. 2005, Sanghvi 2009) that enables distinction between rapid unrestricted diffusion and restricted diffusion. Lesions that have restricted diffusion appear hyper-intense on diffusion images and hypo-intense on the accompanying apparent diffusion coefficient maps. Maps of the apparent diffusion coefficient are generated from different regions scanned. A region of lower diffusion coefficient is more likely to contain more tumor than a high coefficient. This method may also help distinguish between malignant and benign lesions in the brain (Rees 2003). The use of the technique permits the assessment of tumor response during RT and regions of poor response may be identified for radiation boosting. Preliminary studies in rectal cancer treated using chemo- and radiation therapies have suggested that apparent diffusion coefficient values may provide indication of tumor response (Einarsdottir et al. 2004). This may be used to optimize treatment during RT and to initiate adjuvant therapy for individual patient. Thoeny HC et al. (2009) assessed a combination of USPIO particles of iron oxide-enhanced and diffusion-weighted (USPIO-DW) MRI and reliably detect pelvic lymph node metastases in normal-sized nodes of bladder and prostate. They found that USPIO-DW-MRI is a fast and accurate method for detecting pelvic lymph node metastases, even in normal-sized nodes.

(b) Dynamic Contrast-Enhanced (DCE) MRI Technique: This technique and diffusion-weighted MRI provide characterization of healthy tissues and tumors (Einarsdottir et al. 2004, Padhani et al. 1999, Padhani et al. 2001). Sequences like ultra-fast volumetric and 3-D cine-mode allow assessment of target volume and organ motions and deformities [Och et al. 1992, Einarsdottir et al. 2004]. Cine-mode acquisitions can demonstrate target tissue motion (Mazzara et al. 2004). DCE MRI based on the vascular dynamics of new and abnormal tumor growth in neo-angiogenesis is another useful technique. The method may be used to differentiate between normal, tumor and irradiated tissues (Einarsdottir et al. 2004). The studied tissues exhibit enhanced tumor signals and the technique can be used to define tumor volume for TP or for postsurgical tumor bed radiation boosting. $T_{2}$-weighted sequences are more sensitive to the vascular phase and thus better display tissue perfusion and blood volume effects (Sugahara et al. 1998). $T_{1}$-weighted sequences are better at detecting contrast agents in extra-vascular to extra-cellular space and thus demonstrate micro-vessel perfusion, permeability and extracellular leakage effects. $T_{2}$ methods are better for evaluating brain tumors like gliomas (Neeman et al. 2001) whereas $T_{1}$ methods have been used more widely in breast, musculoskeletal, gynaecological and urological cancers (Dao et al. 1993, De Vries et al. 2000, Mayr et al. 2000). These methods have been used to detect tumor recurrence in previously 
irradiated breast (Dao et al. 1993), prostate cancer sites (De Vries et al. 2000, Rouviere et al. 2004) to predict response to RT of head and neck (Tomura et al. 2005), rectal (Dao et al. 1993) and cervical cancers (De Vries et al. 2000).

(c) MRI-guided Radiotherapy (MRIGRT): The fact that normal and abnormal tissues respond differently to slight alterations allows visualizing different types of abnormalities and disease processes better than without contrasts. Temporal-spatial information from MRI can be used for image-guided radiotherapy (IGRT). Integration of $1.5 \mathrm{~T}$ MRI scanner with a $6 \mathrm{MV}$ accelerator has recently been designed and tested by Raaymakers et al. (2009). They achieved this by modification of the two systems to achieve simultaneous and unhindered normal operations of both. This innovation opens the door towards a clinical prototype MRI-guided RT in the routine clinics.

(d) 3-D Real Time in-vivo Magnetic Particle Imaging: Magnetic particle imaging (MPI) is a new tomographic imaging method potentially capable of rapid 3-D dynamic imaging of magnetic tracer materials. Until now only dynamic 2-D phantom experiments with high tracer concentrations have been demonstrated. Weizenecker et al. (2009) recently reported the first in-vivo 3-D real time MPI scans revealing details of a beating mouse heart using contrast agent. They obtained a resolution of $21.5 \mathrm{~ms}$ at a 3-D field of view of $20.4 \times 12 \times 16.8$ $\mathrm{mm}^{3}$ with a spatial resolution sufficient to resolve all the heart chambers.

(e) Ultra-fast BOLD fMRI: Imaging parameters chosen for $\mathrm{fMRI}$ influence the precision of functional localization, affects the image quality and the degree of BOLD contrast achieved. fMRI demonstrates brain function with neuro-anatomical localization on a real time basis. Quantitative assessment allows functional tumor parameters to be calculated using models (Ten Haken et al 1992). Most of such studies were performed using BOLD contrast which requires the detection of very small changes in signal intensities (0 - $3 \%$ ) with $1.5 \mathrm{~T}$ and up to $6 \%$ with $3 \mathrm{~T}$ for voxel volumes as small as $3 \times 3 \times 5 \mathrm{~mm}^{3}$ (Thulborn 2002). Ultrafast BOLD fMRI using single-shot spin-echo planar imaging has recently been announced by Boujraf et al. (2009). Functional imaging techniques have been introduced, using intrinsic contrast mechanisms such as BOLD approach for brain activation (Einarsdottir et al. 2004, Chung et al. 2004), and water diffusion to provide maps of apparent diffusion coefficients discussed earlier.
Brain activation (De Vries et al. 2000, Bondiau et al. 2005) and tractography (Levivier et al. 2004) improve definition of CNS target volumes and sparing of critical functions. Contrast agents and the BOLD techniques may provide information on tissue oxygenation (Manavis et al. 2005) and MR based hypoxic markers are also being evaluated (Boujraf 2009). Extrinsic contrast agents can provide information on tumor properties, and modeling techniques can derive certain range of functional parameters (Chung et al. 2004).

\section{MRI IN POST TREATMENT FOLLOW-UP}

Contrast agents have enabled imaging of biological processes in-vivo and this provides new techniques for monitoring and diagnosis of diseases (Emami et al. 2003). The newly emerging MRI contrast materials present a promising tomorrow in post RT patient monitoring and individualization of procedures. Prominent among these are the USPIO particles. USPIO preparation small enough to migrate across the capillary wall has been developed, which is a prerequisite in the design of targetable particulate pharmaceuticals (Weissleder et al. 1990). The promissory uses of USPIO include as bone marrow contrast agent, intravenous contrast agent for lymphatic system, long half-life perfusion agent for brain, heart and for magnetic moiety in organtargeted superparamagnetic contrast (London, Royal College of Radiologists, 2004). Post RT patient monitoring applications include:

(a) Angiogenesis targeting: This is essential for the development of malignant tumors and provides important targets for tumor diagnosis and therapy (Khoo et al. 1997, Corot et al. 2004, Peng et al. 2008). To non-invasively assess the angiogenic profile of tumors, alpha(v)beta(3) integrin-targeted USPIO particles were designed and their specific uptake by endothelial cells was evaluated in-vitro and in-vivo. The long blood circulating time and the progressive macrophage uptake in inflammatory tissues of USPIO particles are the two properties of major importance for MRI pathologic tissue characterization. Corot et al. (2009) have reviewed the imaging of carotid atherosclerotic plaque, stroke, brain tumor characterization, or multiple sclerosis.

(b) Brain and CNS tumors: USPIO particles which do not pass the ruptured blood-brain barrier at early times post-injection can be used to assess tumoral microvascular heterogeneity (Weissleder et al. 1990, Neumaier et al. 2008). $24 \mathrm{~h}$ after injection, the USPIO tumoral contrast enhancement is higher in high-grade 
than in low-grade tumors. Several experimental studies and a pilot multiple sclerosis clinical trial in 10 patients have shown that USPIO contrast agents can reveal the presence of inflammatory multiple sclerosis lesions (Corot et al. 2004, Corot et al. 2009). The enhancement with USPIO does not completely overlap with the gadolinium chelate enhancement. The fact that USPIO can visualize macrophage infiltrations has been confirmed in animals and patients in several applications like carotid atherosclerotic lesions, stroke, brain tumors and multiple sclerosis.

(c) Tumor targeting: Magnetic iron oxide (MIO) nanoparticles with a long blood retention time, biodegradability and low toxicity have emerged as primary materials for in-vitro and in-vivo applications (Neumaier et al. 2008). MIO nanoparticles have a large surface area and may be modified to provide a large number of functional groups for cross-linking to tumor-targeting ligands like monoclonal antibodies, peptides, or small molecules for diagnostic imaging or delivery of therapeutic agents. MIO nanoparticles possess unique paramagnetic properties, which generate significant susceptibility with strong $T_{2}$ and $\mathrm{T}_{2}{ }^{*}$ contrast, as well as $\mathrm{T}_{1}$ effects at very low concentrations. Real-time image guidance in RT may be used to ramp tumour targeting accuracy and enable real-time tracking of moving targets according to the Cross Cancer Institute research team in Canada (Thornton et al. 1992).

(d) Detection and labeling of tumor cells: Nanoparticles exhibit unique properties of superparamagnetism and can be utilized as probes for MRI. USPIO nanoparticles are suitable for labeling molecular probes that target specific tumorassociated markers for in-vitro and in-vivo detection. Neumaier et al. (2008) demonstrated that $1.5 \mathrm{~T} \mathrm{MRI}$ allows the detection of USPIO nanoparticle conjugated antibody specifically bound to tumor cells in-vitro and in-vivo, and that the MR signal intensity correlates with the concentration of USPIO nanoparticle antibody used and with the antigen density at the cell surface. The product can potentially aid in early disease detection and monitoring of RT efficacy.

(e) Identification of neural stem and progenitor cells: Cell metabolism can be monitored using MRS where either single voxels, 2-D or 3-D metabolic maps are obtained (Heather et al. 2006). The metabolic biomarker for the detection and quantification of neural progenitor cells in the brain invivo described by Manganas et al. (2007) uses MRS for identifying and characterizing the biomarker in which neural progenitor cells were enriched. They demonstrated its use as a reference for monitoring neurogenesis. Identification of neural stem and progenitor cells has important diagnostic, prognostic and therapeutic implications.

\section{CONCLUSION}

Although MRI scans are ideal for diagnosing and evaluating a number of conditions, they do have drawbacks. Patients who wear pacemakers, orthopedic hardware such as screws, plates, artificial joints in the region scanned can cause severe distortions on the image. Some patients are claustrophobic and being in an MRI machine is a disconcerting experience. MRI scans require patients to remain immobile for an extended period of time. The procedures vary in duration from say 20 to 90 min or even longer. The hardware causes a significant alteration in the main magnetic field. MRI systems are very expensive, hence the examinations. There is patient safety concern due to energy deposited using $3 \mathrm{~T}$ scanners which may be up to 4 times that of $1.5 \mathrm{~T}$ machines, especially with fast or intensive pulse sequences. Poor image of bones and the lack of electron density indication required for dosimetry calculations is a problem. Dosimetry using MRI parameters is yet impossible unlike the use of Hounsfield number with CT (Evans 2005). However, studies are going on to possibly overcome this through the use of magnetization gel detectors (Heather et al. 2006).

\section{REFERENCES}

American Association of Physicists in Medicine, Quality Assurance. Methods and Phantoms for Magnetic Resonance Imaging. AAPM, Maryland 1990.

Awojoyogbe O. B., Boubakker K., Aweda M. A. and Dada M. BPES-Related Mathematical Development for the Phase Shift Due to RF Magnetic Field in Heart Inferior Coronary Artery NMR Imaging. J Clinic Experiment Cardiol. 1(2) Available at: http://www.omicsonline.org/2155-9880/2155-9880-1111.php

Awojoyogbe O. B., Dada M., Faromika O. P., Aweda M. A. and Fuwape I. A. Mathematical formulation of NMR experimental parameters for diffusion magnetic resonance imaging - part II (Cylindrical geometry) Intern'l J.Maths, Game Theory and Algebra. 20(1); 2040. (2010). Available at:

https://www.novapublishers.com/catalog/product_info. php?products_id=18401 
Barentsz J. O., Engelbrecht M. R., Witjes J.A., de la Rosette J. J., van der Graaf M. MR imaging of the male pelvis. Eur Radiol. 9:1722-1736. (1999).

Bloch B. N., Rofsky N. M., Baroni R. H., Marquis R. P., Pedrosa I. and Lenkinski R. E. 3 Tesla magnetic resonance imaging of the prostate with combined pelvic phased-array and endorectal coils; Initial experience(1). Acad. Radiol. 11:863-867. (2004)

Board of the Faculty of Clinical-Oncology Document on Imaging for oncologists: collaboration between clinical radiologists and clinical oncologists in diagnosis, staging and radiotherapy planning. London, Royal College of Radiologists. (2004).

Bondiau P. Y., Malandain G., Chanalet S, Marcy P. Y., Habrand J. L. and Fauchon F. Atlas-based automatic segmentation of MR images: validation study on the brainstem in radiotherapy context. Int J. Radiat Oncol Biol Phys. 61:289-298. (2005)

Chao Wang, Shuang Luan, Grace Tang, Danny Z. Chen, Matt A. Earl, Cedric X. Yu. Arc-modulated radiation therapy (AMRT): a single-arc form of intensitymodulated arc therapy. Phys Med Biol. 53:6291-303. (2008)

Chung N. N., Ting L. L., Hsu W. C., Lui L. T. Wang P. M. Impact of magnetic resonance imaging versus $\mathrm{CT}$ on nasopharyngeal carcinoma: primary tumor target delineation for radiotherapy. Head Neck. 26:241-246. (2004)

Corot C., Petry K. G, Trivedi R., Saleh A., Jonkmanns C., Le Bas J. F. Macrophage imaging in central nervous system and in carotid atherosclerotic plaque using ultrasmall superparamagnetic iron oxide in magnetic resonance imaging. Invest Radiol. 39:619-625. (2004)

Dada M., Faromika O. P., Awojoyogbe O. B., Aweda M. A. and Fuwape I. A. Mathematical formulation of NMR experimental parameters for diffusion magnetic resonance imaging - part I (Spherical geometry) Int'l J.Maths, Game Theory and Algebra. 20(1); 1-19. (2010). https://www.novapublishers.com/catalog/product_info. php?products_id=18401

Damadian R., Goldsmith M., Minkoff L. NMR in cancer: $\mathrm{XVI}$. Fonar image of the live human body. Physiol Chem Phys. 9:97-100. (1977)

Dao T. H., Rahmouni A., Campana F., Laurent M., Asselain B., Fourquet A. Tumor recurrence versus fibrosis in the irradiated breast: differentiation with dynamic gadolinium- enhanced MR imaging. Radiology. 187:751-5. (1993)

De Vries A., Griebel J., Kremser C., Judmaier W., Gneiting T., Debbage $P$. Monitoring of tumor microcirculation during fractionated radiation therapy in patients with rectal carcinoma: preliminary results and implications for therapy. Radiology. 217: 385-391. (2000)
Dzik-Jurasz A., Domenig C., George M., Wolber J., Padhani A., Brown G. Diffusion MRI for prediction of response of rectal cancer to chemoradiation. Lancet. 360:307-308. (2002)

Einarsdottir H., Karlsson M., Wejde J., Bauer H. C. Diffusion weighted MRI of soft tissue tumours. Eur Radiol. 14:959-63. (2004)

Emami B, Sethi A, Petruzzelli G. J. Influence of MRI on target volume delineation and IMRT planning in nasopharyngeal carcinoma. Int $\mathrm{J}$ Radiat Oncol Biol Phys. 57:481-8. (2003)

Evans M. C. D. Computerized treatment planning systems for external photon beam radiotherapy. In, Podgorsak EB. (Ed). Radiation Oncology Physics: A handbook for teachers and students. Vienna, International Atomic Energy Agency, 387-406. (2005)

Gregoire V, Coche E, Cosnard G, Hamoir M, Reychler H. Selection and delineation of lymph node target volumes in head and neck conformal radiotherapy: Proposal for standardizing terminology and procedure based on the surgical experience. Radiother Oncol. 56:135-50. (2000)

Harisinghani M. G., Saini S, Weissleder R, Hahn P. F., Yantiss R. K., Tempany C. M. R. Lymphangiography using ultrasmall superparamagnetic iron oxide in patients with primary abdominal and pelvic malignancies: radiographic-pathologic correlation. Am J Roentgenol. 172:1347-1351. (1999)

Heather M, Whitney, Daniel F, Gochberg, John C, Gore. Magnetization transfer in polymer gel dosimeters. 4th International Conference on Radiotherapy Gel Dosimetry. Conference Series Institute of Physics Publishing Journal of Physics, 56:253-255. (2006)

Heester M. A, Wijrdeman H. K, Strukmans H., Witkamp. T, Moerland M. A. Brain tumor delineation based on CT and MR imaging. Strahlenther Onkol. 169:729-33. (1993)

Hinshaw D. S., Bottomley P. A. and Holland G. N. Radiographic thin-section image of the human wrist by nuclear magnetic resonance. Nature. 270:722-23. (1977)

Huerlimann MD, Carr-Purcell sequences with composite pulses. J. Magn Reson. 152:109-23. (2001)

Hrabe J., Gurjinder Kaur, David N. Guilfoyle. Principles and limitations of NMR diffusion measurements. Journ Med Phys. 32:34-42. (2007)

IPEM Report 80: Quality control in magnetic resonance imaging. IPEM, York (1995).

Khoo V. S. MRI Magic radiotherapy imaging for treatment planning? Br J Radiol 73:229-33. (2000) 
Khoo V. S., Adams E. J., Saran F, Bedford J. L. Perks J. R. Warrington A. P. A comparison of clinical target volumes determined by CT and MRI for the radiotherapy planning of base of skull meningiomas. Int J Radiat Oncol Biol Phys. 46:1309-1317. (2000)

Khoo V. S., Dearnaley D. P, Finnigan D. J, Padhani A, Tanner S. F, Leach M. O. Magnetic resonance imaging (MRI): considerations and applications in radiotherapy treatment planning. Radiother Oncol. 42:1-15. (1997)

Khoo V. S, Dearnaley D. P, Finnigan D, J, Padhani A, Tanner S. F, Leach M. O. Magnetic resonance imaging (MRI): considerations and applications in radiotherapy treatment planning. Cancer Imaging 22:100-1. (2006)

Khoo V. S, Padhani A. R, Tanner S. F, Finnigan D. J, Leach M. O, Dearnaley D. P. Comparison of MRI with CT for the radiotherapy planning of prostate cancer: a feasibility study. Br J Radiol. 72:590-597 (1999).

Krauss D. J., Kestin L. L., Raff G, Yan D, Wong J, Gentry R. MRI-based volumetric assessment of cardiac anatomy and dose reduction via active breathing control during irradiation for left-sided breast cancer. Int J Radiat Oncol Biol Phys. 61:1243-50. (2005)

Krempien R. C, Daeuber S, Hensley F. W, Wannenmacher $\mathrm{M}$, Harms W. Image fusion of $\mathrm{CT}$ and MRI data enables improved target volume definition in 3Dbrachytherapy treatment planning. Brachytherapy. 2:164-71. (2003)

Kremser C, Judmaier W, Hein P, Griebel J, Lukas P, de Vries A. Preliminary results on the influence of chemoradiation on apparent diffusion coefficients of primary rectal carcinoma measured by magnetic resonance imaging. Strahlenther Onkol. 179:641-649. (2003)

Le Bihan D, Breton E, Lallemand D, Grenier P, Cabanis E, Laval-Jeantet M. MR imaging of intravoxel incoherent motions: Application to diffusion and perfusion in neurologic disorders. Radiolog. 161:401-407. (1986)

Lee Y. K, Bollet M, Charles-Edwards G, Flower M. A, Leach M. O. et al. Radiotherapy treatment planning of prostate cancer using magnetic resonance imaging alone. Radiother Oncol. 66:203-16. (2000)

Levivier M, Massager N, Wikler D, Goldman S. Modern multimodal neuroimaging for radiosurgery: the example of PET scan integration. Acta Neurochir. 91:1-7. (2004)

Li X, Du X, Huo T, Liu X, Zhang S, Yuan F. Specific targeting of breast tumor by octreotide-conjugated ultrasmall superparamagnetic iron oxide particles using a clinical 3.0-Tesla magnetic resonance scanner. Acta Radiol 50(6):583-594. (2009)

Liu H. H, Koch N, Starkschall G, Jacobson M, Forster K, Liao Z. Evaluation of internal lung motion for respiratorygated radiotherapy using MRI: Part IImargin reduction of internal target volume. Int J Radiat Oncol Biol Phys. 60:1473-83. (2004)

Los Alamos National Laboratory. MEG and MRI: getting it together? [Internet Uploaded 2007 January 31; cited 2009 April 10] Available at http://medicalphysicsweb.org/cws/article/research/269 43

Mack A, Wolff R, Scheib S, Rieker M, Weltz D, Mack G. Analyzing 3-tesla magnetic resonance imaging units for implementation in radiosurgery. J Neurosurg 102:58-64. (2005)

Mack M. G, Balzer J. O, Straub R, Eichler K, Vogl T. J. Super-paramagnetic iron oxide-enhanced MR imaging of head and neck lymph nodes. Radiology 222:239-44. (2002)

Mackenzie R, Dixon A. K. Measuring the effects of imaging: an evaluative framework. Clin Radiol 50:513-8. (1995)

Mah K, Caldwell C, Ung Y, Danjoux C, Balogh J, Ganguli $N$. Characteristics and quality assurance of a dedicated open $0.23 \mathrm{~T}$ MRI for radiation therapy simulation. Med Phys. 29:2541-2547. (2002)

Manavis J, Sivridis L, Koukourakis M. I. Nasopharyngeal carcinoma: the impact of CT-scan and of MRI on staging, radiotherapy treatment planning, and outcome of the disease. Clin Imaging. 29:128-133. (2005)

Manganas L. N, Xueying Zhang, Yao Li, Raphael D. Hazel, S. David Smith, Mark E. Wagshul, et al. Magnetic Resonance Spectroscopy Identifies Neural Progenitor Cells in the Live Human Brain. Science. 318:980 - 985. (2007)

Martin J, Joon D. L, Ng N, Grace M, Van-Gelderen D, Lawlor M. Towards individualised radiotherapy for stage I seminoma. Radiother Oncol. 76:251-6. (2005)

Mayr N. A, Yuh W. T, Arnholt J. C, Ehrhardt J. C, Sorosky J. I, Magnotta V. A. Pixel analysis of MR perfusion imaging in predicting radiation therapy outcome in cervical cancer. J Magn Reson Imaging 12:1027-33. (2000)

Mazzara G. P, Velthuizen R. P, Pearlman J. L, Greenberg $\mathrm{H}$. $\mathrm{M}$, Wagner $\mathrm{H}$. Brain tumor target volume determination for radiation treatment planning through automated MRI segmentation. Int J Radiat Oncol Biol Phys. 59:300-12. (2004)

Mizowaki T, Nagata Y, Okajima K, Murata R, Yamamoto M, Kokubo $\mathrm{M}$, et al. Development of an MR simulator: experimental verification of geometric distortion and clinical application. Radiology 199:855-60. (1996)

Mizowaki T, Cohen G. N., Fung A. Y., Zaider M. Towards integrating functional imaging in the treatment of prostate cancer with radiation: the registration of the Magnetic Resonance spectroscopy imaging to 
ultrasound/CT images and its implementation in treatment planning. Int $\mathrm{J}$ Radiat Oncol. Biol Phys. 54:1558-64. (2002)

Neeman M, Provenzale J. M, Dewhirst M. W. Magnetic resonance imaging applications in the evaluation of tumor angiogenesis. Semin Radiat Oncol. 11:70-82. (2001)

Neumaier CE, Baio G, Ferrini S, Corte G, Daga A. MR and iron magnetic nanoparticles. Tumori. 94:226-33. (2008)

Newbold K, Partridge M, Cook G, Sohaib S. A, CharlesEdwards E, Rhys-Evans P. Advanced imaging applied to radiotherapy planning in head and neck cancer: a clinical

Br J Radiol. 79:554-61. (2006)

review.

Och J. G, Clarke G. D, Sobol W. T, Rosen C. W, Mun S. K. Acceptance testing of magnetic resonance imaging systems: report of AAPM Nuclear Magnetic Resonance Task Group No. 6. Med Phys 19:217-29. (1992)

Padhani A. R., Husband J. E. Dynamic contrast-enhanced MRI studies in oncology with an emphasis on quantification, validation and human studies. Clin Radiol. 56:607-20. (2001)

Padhani A. R., Khoo V. S., Suckling J., Husband J. E., Leach M. O. Dearnaley D. P. Evaluating the effect of rectal distension and rectal movement on prostate gland position using cine MRI. Int J Radiat Oncol Biol Phys. 44:525-33. (1999)

Parke W. and Patrocinio $\mathrm{H}$. Clinical treatment planning in external photon beam radiotherapy. In, Podgorsak EB. (Ed). Radiation Oncology Physics: A handbook for teachers and students. Vienna, International Atomic Energy Agency. 219-272. (2005)

Partridge M., Trapp J. V., Adams E. J., Leach M. O., Webb S., Seco J. An investigation of dose calculation accuracy in intensity-modulated radiotherapy of sites in the head and neck. Phys Med 22:97-104. (2006)

Payne G. S. and Leach M. O. Applications of magnetic resonance spectroscopy in radiotherapy treatment planning, Br J Radiol. 79:16-26. (2006)

Pech M, Mohnike K, Wieners G, Bialek E, Dudeck O, Seidensticker M. Radiotherapy of liver metastases. Comparison of target volumes and dose-volume histograms employing CT- or MRI-based treatment planning. Strahlenther Onkol. 184:256-61. (2008)

Peng XH, Qian X, Mao H, Wang AY, Chen ZG, Nie S. Targeted magnetic iron oxide nanoparticles for tumor imaging and therapy. Int $\mathrm{J}$ Nanomedicine. 3:311-21. (2008)

Pickett B., Vigneault E., Kurhanewicz J., Verhey L., Roach M. Static field intensity modulation to treat a dominant intraprostatic lesion to 90 Gy compared to seven field 3-dimensional radiotherapy. Int J Radiat Oncol Biol Phys. 44:921-9. (1999)

Plathow C., Ley S., Fink C., Puderbach M., Hosch W. A. Analysis of intrathoracic tumor mobility during whole breathing cycle by dynamic MRI. Int J Radiat Oncol Biol Phys 59:952-9 (2004)

Plathow C, Ley S, Fink C, Puderbach M, Hosch W. Schmahl A. Analysis of intrathoracic tumor mobility during whole breathing cycle by dynamic MRI. Int J Radiat Oncol Biol Phys 59:952-959. (2004)

Podgorsak EB, Podgorsak MB. Special procedures and techniques in radiotherapy. In, Podgorsak EB. (Ed). Radiation Oncology Physics: A handbook for teachers and students. Vienna, International Atomic Energy Agency, 2005:505-548.

Portaluri M, Bambace S, Perez C, Giuliano G, Angone G, Scialpi M. Clinical and anatomical guidelines in pelvic cancer contouring for radiotherapy treatment planning. Cancer Radiother. 8:222-9. (2004)

Price R. R., Axel L., Morgan T., Newman R., Perman W., Schneiders N. Quality assurance methods and phantoms for magnetic resonance imaging: report of AAPM nuclear magnetic resonance Task Group No 1. Med Phys. 17:287-95. (1990)

Price S. J, Burnet N. G, Donovan T, Green H. A, Pena A, Antoun N. M. Diffusion tensor imaging of brain tumours at 3T: a potential tool for assessing white matter tract invasion? Clin Radiol 58:455-62 (2003)

Raaymakers B. W. Lagendijk J. J., Overweg J, Kok J. G. M., Raaijmakers A. J. E., Kerkhof E. M. Integrating a 1.5 T MRI scanner with a $6 \mathrm{MV}$ accelerator: proof of concept. Phys Med Biol 54:229-37. (2009)

Rasch C, Barillot I, Remeijer P, Touw A, van Herk M, Lebesque J. V. Definition of the prostate in $\mathrm{CT}$ and MRI: a multi-observer study. Int $\mathrm{J}$ Radiat Oncol Biol Phys. 43:57-66. (1999)

Rees J. Advances in magnetic resonance imaging of brain tumours. Curr Opin Neurol 16:643-50. (2003)

Rohlfing T, Maurer C. R. Jr, O'Dell W. G., Zhong J. Modeling liver motion and deformation during the respiratory cycle using intensity-based nonrigid registration of gated MR images. Med Phys. 31:42732. (2004)

Rosen $Y$. The Recent advances in magnetic resonance neurospectroscopy. Neurotherapeutics. 27:330-45. (2007)

Rouviere O, Valette O, Grivolat S, Colin-Pangaud C, Bouvier R, Chapelon J. Y. Recurrent prostate cancer after external beam radiotherapy: value of contrastenhanced dynamic MRI in localizing intraprostatic 
tumor--correlation with biopsy findings. Urology. 63:922-7. (2004)

Said Boujraf, Paul Summers, Faouzi Belahsen, Klaas Prussmann, Spyros Kollias. Ultrafast bold fMRI using single-shot spin-echo echo planar imaging. J Med Phys. 34:37-42. (2009)

Sanghvi DA. Recent advances in imaging of brain tumors. Indian J. Cancer. 46:82-7. (2009)

Seddon B. M, Payne G. S, Simmons L, Ruddle R, Grimshaw R, Tan S. A phase I study of SR-4554 via intravenous administration for noninvasive investigation of tumor hypoxia by magnetic resonance spectroscopy in patients with malignancy. Clin Cancer Res. 9:5101-2. (2003)

Solberg T. D., Agazaryan N, Goss B. W., Dahlbom M., Lee S. P. A feasibility study of 18F-fluorodeoxyglucose positron emission tomography targeting and simultaneous integrated boost for intensity-modulated radiosurgery and radiotherapy. J Neurosurg. 101:3819. (2004)

Sosna J, Pedrosa I, Dewolf W. C, Mahallati H, Lenkinski R. E, Rofsky N. M. MR imaging of the prostate at 3 Tesla: comparison of an external phased-array coil to imaging with an endorectal coil at 1.5 Tesla. Acad Radiol. 11:857-62. (2004)

Sugahara T, Korogi Y, Kochi M, Ikushima I, Hirai T, Okuda $\mathrm{T}$. Correlation of MR imaging-determined cerebral blood volume maps with histologic and angiographic determination of vascularity of gliomas. Am J Roentgenol. 171:1479-86. (1998)

Sultanem K, Patrocinio H, Lambert C, Corns R, Leblanc R, Parker W. The use of hypofractionated intensity modulated irradiation in the treatment of glioblastoma multiforme: preliminary results of a prospective trial. Int J Radiat Oncol Biol Phys. 58:247-52. (2004)

Ten Haken R. K, Thornton A. F, Sandler H. M, La Vigne M. L, Quint D. J, Frass B. A. A quantitative assessment of the addition of MRI to CT-based, 3-D treatment planning of brain tumors. Radiother Oncol. 25:121-33. (1992)

Thoeny H. C, Triantafyllou M, Birkhaeuser F. D, Froehlich J. M, Tshering D. W, Binser T. Eur Urol. 55:761-9. (2009)

Thomas Bortfeld, Steve Webb. Single-Arc IMRT? Phys Med Biol. 54:9-20. (2009)

Thornton A. F, Sandler H. M, Ten Haken R. K, McSchan D. L, Fraass B. A, La Vigne M. L. The clinical utility of magnetic resonance imaging in the 3-dimensional treatment planning of brain tumors. Int J Radiat Oncol Biol Phys. 24:767-75. (1992)

Thulborn K. R. Clinical functional MR imaging. In: Atlas SW, editor. MRI of the brain and spine. 3rd edition. Philadelphia: Lipincott Williams and Wilkins; 2002.

Tomura N, Omachi K, Sakuma I, Takahashi S, Izumi J, Watanabe O. Dynamic contrast enhanced magnetic resonance imaging in radiotherapeutic efficacy in the head and neck tumors. Am J Otolaryngol. 26:163-7. (2005)

Watanabe Y, Akimitsu T, Hirokawa Y, Mooij R. B, Perera G. M. Evaluation of dose delivery accuracy of Gamma Knife by polymer gel dosimetry. J Appl Clin Med Phys 6:133-42. (2005)

Weissleder R, Elizondo G, Wittenberg J, Rabito CA, Bengele $\mathrm{HH}$, Josephson $\mathrm{L}$. Ultrasmall superparamagnetic iron oxide: characterization of a new class of contrast agents for MR imaging. Radiology 175:489-93. (1990)

Weizenecker J, Gleich B, Rahmer J, Dahnke H. Borgert J. 3-D real-time in-vivo magnetic particle imaging. Phys Med Biol. 54:1-10. (2009)

Zhang C, Jugold M, Woenne E. C, Lammers T, Morgenstern B, Mueller M. M. Specific targeting of tumor angiogenesis by RGD-conjugated ultrasmall superparamagnetic iron oxide particles using a clinical 1.5-T magnetic resonance scanner. Cancer Res. 67:1555-62. (2007) 